\section{Regional cardiac resuscitation systems of care}

\author{
NICHOL GRAHAM
}

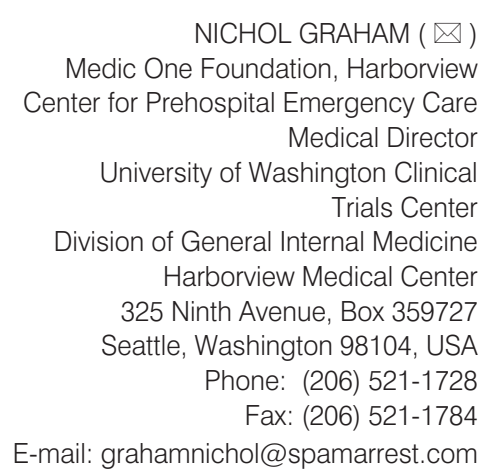

NICHOL GRAHAM $(\bowtie)$ Medic One Foundation, Harborview Center for Prehospital Emergency Care Medical Director University of Washington Clinical Trials Center Division of General Internal Medicine Harborview Medical Center 325 Ninth Avenue, Box 359727 Seattle, Washington 98104, USA Phone: (206) 521-1728 Fax: (206) 521-1784

E-mail: grahamnichol@spamarrest.com

\begin{abstract}
Out-of-hospital cardiac arrest (OOHCA) is a common public health problem, with large and important regional variations in outcomes. Survival rates vary widely among patients treated with OOHCA by emergency medical services (EMS), or among patients transported to the hospital after return of spontaneous circulation. Most regions lack a well-coordinated approach to post-cardiac arrest care. Effective hospital-based interventions for OOHCA exist but are used infrequently. Increased volume of patients or procedures of individual providers and hospitals is associated with better outcomes for several other clinical disorders. Regional systems of cardiac resuscitation include a process for identification of patients with OOHCA, standard field and hospital care protocols for patients with OOHCA, monitoring of care processes and outcome, and periodic review and feedback of these quality improvement data to identify problems and implement solutions. Similar systems have improved provider experience and patient outcomes for those with ST-elevation myocardial infarction and life-threatening traumatic injury. Many more people could survive OOHCA if regional systems of cardiac resuscitation were implemented and maintained. The time has come to do so wherever feasible.
\end{abstract}

Key words: out-of-hospital cardiac arrest, emergency medical services, hospital-based interventions, regional systems of cardiopulmonary resuscitation (CPR), monitoring, outcome, transport time, improve of quality, survival

\section{Background}

Out-of-hospital cardiac arrest (OOHCA) is a common public health problem that affects 235,000 to 325,000 people in the United States, (1) or 275,000 persons individuals in Europe each year. (2) There is a large variation in survival after emergency medical services (EMS) -treated OOHCA (1) as well as after admission following successful resuscitation from OOHCA. (3-5) Such differences are not explained by differences in patient characteristics, (6) so variation in hospital-based care contributes to differences in outcomes across hospitals. But few communities have achieved sustained improvements in survival after cardiac resuscitation. (7-9) In contrast to this lack of improvement in survival over time, implementation of regional systems of care for those with traumatic injury (10) and acute myocardial infarction (AMI) (11-13) improved outcomes for patients with these conditions. Therefore development and implementation of cardiac resuscitation systems of care are recommended to try to achieve similar improvements in outcomes. (14) Regional systems of cardiac resuscitation include a process for identification of patients with OOHCA, standard field and hospital care protocols for patients with $\mathrm{OOHCA}$, monitoring of care processes and outcome, and periodic review and feedback of these quali- ty improvement data to identify problems and implement solutions. Such regionalization of care should improve implementation of intra-arrest and post-arrest interventions for patients who receive care in these systems. But expert recommendation of regionalized resuscitation care does not guarantee its adoption into clinical practice. (15) Some regions have attempted to implement such systems by transporting patients resuscitated in the field from OOHCA only to hospitals capable of inducing hypothermia. (16) But other regions have been unable to do so. (17) Cardiac resuscitation systems of care have not been formally evaluated so there is no direct evidence of the impact of such programmatic interventions on the structure, process, or outcome of resuscitation. Here we summarize evidence of the effectiveness of cardiac 
resuscitation systems, and extrapolate from evidence of the effectiveness of systems of care for other related disorders to cardiac resuscitation.

\section{Transport time}

An interesting issue is how outcomes are related to the duration of transport time if patients resuscitated from cardiac arrest are to be preferentially transported to designated receiving hospitals. In a single urban American EMS system during the pre-hypothermia era, the median transport time was 7 (interquartile range, IQR 5, 11) minutes. (18) The duration of transport to hospital was not significantly associated with survival to hospital discharge after OOHCA. (18)

In a multicenter observational study in Ontario during the pre-hypothermia era, the median transport interval was 4.2 (IQR, 3.0, 6.2) minutes. (19) Duration of transport was not significantly associated with survival. But interpretation of this study is difficult because $19 \%$ of the time interval data were missing, and overall survival was low.

In a multicenter observational study in Arizona during 2004 to 2006, the median transport time was 6 (interquartile range, IQR 4, 9). (20) The duration of transport to hospital was not significantly associated with survival to discharge among patients with restoration of circulation in the field after OOHCA. But interpretation of this study is difficult because transport time data were missing in $38 \%$ of cases.

In another multicenter observational study of patients with OOHCA in North America, overall mean difference in transport distance for patients taken to distant hospital compared to the closest hospital was small [1.70 miles (2.74 km)]. (21) For patients with a difference greater than 5 miles $(N=151)$, unadjusted survival to discharge was lower among those taken to the closest hospital compared to those transported to distant hospitals $(12.1 \%$ vs. $16.5 \%, p<0.001)$. But interpretation of this study is difficult because it did not measure which hospital-based interventions patients received although the further hospitals were more likely to have percutaneous coronary intervention $(\mathrm{PCl})$ facilities, electrophysiology laboratories, more beds, higher patient volumes and teaching hospital status. Collectively these studies suggest that it is feasible to bypass a hospital after circulation has been restored but do not define how long a safe journey time is. Relationship between characteristics of receiving hospital and outcome

An observational study in Osaka Japan, described outcomes after OOHCA in the period between 2005 and 2007. (22) Transfer to a 'critical care centre' was associated with increased good neurological survival at one-month (193 [6.7\%] vs. 213 [2.8\%] P < 0.001) compared to a 'non critical care centre'. The overall improvement in outcomes for patients transferred to 'critical care centres' was due to greater survival of patients who arrived without return of spontaneous circulation (ROSC). This study was difficult to interpret as it did not describe how care process differed between patients.

Case-control studies have evaluated the effectiveness of combinations of hospital-based treatments in patients resuscitated from cardiac arrest in a variety of settings. (1-6) All of these studies have reported improved outcomes when compared with historical controls. These studies are difficult to interpret due to heterogeneity of intervention across studies and because of potential for Hawthorne effects during the intervention period. But collectively these studies suggest that organized approaches to post-resuscitation care in hospital improve outcomes after OHCA.

\section{Relationship between case volume and outcome}

The concept of cardiac resuscitation systems of care is supported by multiple examples throughout the field of medicine of the association between greater provider experience or procedural volume for complex diagnoses or procedures and better patient outcome. (23) The relationship between volume and outcome is complex. Pro- cedural volume is an identifiable surrogate marker for a number of patient, physician, hospital and systems variables that have an impact on outcome but are difficult to quantify individually. The beneficial effect of volume on patient outcome is noted in health conditions that involve a systems-based approach. These include the care of patients with conditions that require time-sensitive intervention, including in-hospital and out-of-hospital cardiac arrest, $(4,24)$ traumatic injury, (25) and patients with ST-elevation myocardial infarction (STEMI) who undergo primary angioplasty, (26) and patients who receive mechanical ventilation for respiratory failure. (27)

Among nine North American regions, 3,644 patients with OOHCA were transported to 254 receiving hospitals. (28) $32 \%$ of patients survived to discharge. Larger hospitals with cardiac catheterization facilities tended to have better patient outcomes compared with no such facilities. Hospitals that received at least 40 patients with cardiac arrest annually tended to have better patient outcomes compared with facilities that received fewer patients. Hospital size, presence of cardiac catheterization facilities and patient volume are inferred to be surrogate markers for better post-resuscitation care.

\section{Trauma systems of care}

Regionalization of care for patients with traumatic injuries has been evaluated in multiple observational studies. (29) Estimation of an overall effect of regionalization of trauma care is difficult because of the heterogeneity of study inclusion criteria, interventions, baseline characteristics of patients, date of study performance, and research design. Collectively these studies demonstrate that implementation of regionalized trauma systems is associated with improved outcome.

\section{STEMI systems of care}

Regionalization of care for patients with AMI (11-13) has led to significant and important improvements in outcome. Pooled analyses of randomized trials 
have demonstrated that primary $\mathrm{PCl}$ reduces rates of death, recurrent myocardial infarction (MI), and hemorrhagic stroke compared with fibrinolysis for patients with acute STEMI. (30) For this reason, use of primary $\mathrm{PCI}$ is recommended in patients with STEMI. (31) But a majority of hospitals lack $\mathrm{PCl}$ facilities that are active 24 hours a day, 7 days a week. For patients who are initially treated at these non-PCl centers, recent observational and randomized trials demonstrate that rapid triage and transport is superior to on-site fibrinolytic therapy. (29) Despite heterogeneity of interventions, settings, and research design, these studies show consistent benefits associated with regionalization of care compared to localized care.

\section{Implementation issues}

Resources in any society are limited, and health care must be allocated efficiently. (32) Some may argue that regionalized resuscitation care is likely to be associated with excessive costs or charges. But resuscitation interventions that increase survival are associated with good quality of life (33-35) at acceptable cost to society. (36)

Regional EMS systems (37) and trauma systems of care (38) have been established throughout the United States with little dedicated funding. In Europe, trauma systems of care are less developed but have similar challenges. (39) In either setting, demand for emergency or trauma care often exceeds the ability of hospitals to provide it. If reimbursements fail to cover emergency department and trauma service costs, these costs are subsidized by revenue from admissions that originated in the emergency department. Such uncompensated care is a burden for hospitals with large numbers of uninsured patients. (40) Trauma centers in the United States lose more than $\$ 1$ billion annually due to a disproportionate and increasing share of patients without the means to pay, lack of ability to shift cost to finance trauma care, difficult relationships with managed care, no payment by Medicare for standby costs, and insufficient reimbursement by auto insurers or state Medicaid programs. Some American hospitals that provided emergency and trauma care have closed in recent years because of financial losses. (41) Even in the presence of universal health insurance, funding arrangements for trauma care do not reflect the true costs incurred by the hospital that treat higher volumes of patients with trauma. (39)

Public and tertiary hospitals bear a large share of this burden because surrounding community hospitals often transfer their most complex, high-risk patients to these safety net hospitals for specialized care. To ensure the continued viability of a critical public safety function, experts have recommended that governments establish dedicated funding to reimburse hospitals that provide significant amounts of uncompensated emergency and trauma care for their associated financial losses. $(39,42)$

Successful implementation and maintenance of cardiac resuscitation systems of care will require increased funding for care provided by EMS, level 2/referring cardiac resuscitation centers, and level $1 /$ receiving cardiac resuscitation centers. Such funding might be made possible through a system of shared reimbursement for systems of care, including payments to smaller referral hospitals that prepare and transfer these complex cases to the receiving hospital, to EMS for providing emergent inter-facility transport with vigilance for rearrest, and to receiving hospitals that ultimately provide the bulk of post-cardiac arrest care. Such shared reimbursement should include provisions for "Pay for Performance." If the system of care delivers better quality of care, then each component of the system should be rewarded.

In summary, the scientific literature related to OOHCA and other acute lifethreatening illnesses supports implementation and maintenance of regional systems of care for those with restoration of circulation after OOHCA; concentrate specialized post-resuscitation skills in selected hospitals; transfer unconscious post-cardiac arrest patients to these hospitals as appropriate; monitor, report, and try to improve cardiac resuscitation structure, process, and outcome; and reimburse these activities. Successful implementation and maintenance of cardiac resuscitation systems of care would have a significant and important impact on a leading global cause of death. The time to implement these systems of care is now. 


\section{REFERENCES}

1. Nichol G, Thomas E, Callaway CW, Hedges J, Powell JL, Aufderheide TP, et al. Regional variation in out-of-hospital cardiac arrest incidence and outcome. JAMA 2008; 300(12):1423-31.

2. Atwood C, Eisenberg MS, Herlitz J, Rea TD. Incidence of EMS-treated out-of-hospital cardiac arrest in Europe. Resuscitation 2005;67(1):7580 .

3. Herlitz J, Engdahl J, Svensson L, Angquist KA, Silfverstolpe J, Holmberg S. Major differences in 1-month survival between hospitals in Sweden among initial survivors of out-of-hospital cardiac arrest. Resuscitation 2006;70(3):404-9.

4. Carr BG, Kahn JM, Merchant RM, Kramer AA, Neumar RW. Inter-hospital variability in post-cardiac arrest mortality. Resuscitation 2009;80(1):30-4

5. Liu JM, Yang Q, Pirrallo RG, Klein JP, Aufderheide TP. Hospital variability of out-of-hospital cardiac arrest survival. Prehosp Emerg Care 2008;12(3):339-46.

6. Rea TD, Cook AJ, Stiell IG, Powell J, Bigham B, Callaway CW, et al. Predicting Survival After Out-of-Hospital Cardiac Arrest: Role of the Utstein Data Elements. Ann Emerg Med 2010;55:249-57.

7. Rea TD, Eisenberg MS, Becker LJ, Murray JA, Hearne T. Temporal trends in sudden cardiac arrest: a 25-year emergency medical services perspective. Circulation 2003;107(22):2780-5.

8. Iwami T, Nichol G, Hiraide A, Hayashi Y, Nishiuchi T, Kajino K, et al. Continuous improvements in "Chain of Survival" increased survival after out-of-hospital cardiac arrests: a large-scale population-based study. Circulation 2009;119(5):728-34.

9. Herlitz J, Bang A, Gunnarsson J, Engdahl J, Karlson BW, Lindqvist J, et al. Factors associated with survival to hospital discharge among patients hospitalised alive after out of hospital cardiac arrest: change in outcome over 20 years in the community of Goteborg, Sweden. Heart 2003;89(1):25-30.

10. MacKenzie EJ, Rivara FP, Jurkovich GJ, Nathens AB, Frey KP, Egleston BL, et al. A national evaluation of the effect of trauma-center care on mortality. N Engl J Med 2006;354(4):366-78.

11. Jacobs AK. Regional systems of care for patients with ST-elevation myocardial infarction: being at the right place at the right time. Circulation 2007;116(7):689-92.

12. Henry TD, Sharkey SW, Burke MN, Chavez IJ, Graham KJ, Henry CR, et al. A regional system to provide timely access to percutaneous coronary intervention for ST-elevation myocardial infarction. Circulation 2007;116(7):721-8.

13. Ting HH, Rihal CS, Gersh BJ, Haro LH, Bjerke CM, Lennon RJ, et al. Regional systems of care to optimize timeliness of reperfusion therapy for ST-elevation myocardial infarction: the Mayo Clinic STEMI Protocol. Circulation 2007;116(7):729-36.

14. Nichol G, Aufderheide TP, Eigel B, Neumar RW, Lurie KG, Bufalino VJ, et al. Regional Systems of Care for Out-of-Hospital Cardiac Arrest: A Policy Statement From the American Heart Association. Circulation 2010;In Press.

15. Siegel D. The gap between knowledge and practice in the treatment and prevention of cardiovascular disease. Prev Cardiol 2000;3(4):16771 .

16. Hartocollis A. City pushes cooling therapy for cardiac arrest New York Times. New York Times December 4,2008;A1.

17. Spector H. MetroHealth, Cleveland EMS don't back regional approach for cardiac arrest therapy. MetroHealth, EMS: not enough evidence to alter treatment. Plain Dealer. Published online March 23, 2009 (cited 2010 July 25). Available: http://www.cleveland.com/medical/plaindealer/index.ssf?/base/news/123779719327900.xml\&coll=2. Accessed January 5, 2010

18. Davis DP, Fisher R, Aguilar S, Metz M, Ochs G, McCallum-Brown L, et al. The feasibility of a regional cardiac arrest receiving system. Resuscitation 2007;74(1):44-51.

19. Spaite DW, Stiell IG, Bobrow BJ, de Boer M, Maloney J, Denninghoff K, et al. Effect of transport interval on out-of-hospital cardiac arrest survival in the OPALS study: implications for triaging patients to specialized cardiac arrest centers. Ann Emerg Med 2009;54(2):248-55.

20. Spaite DW, Bobrow BJ, Vadeboncoeur TF, Chikani V, Clark L, Mullins T, et al. The impact of prehospital transport interval on survival in out-of-hospital cardiac arrest: implications for regionalization of post-resuscitation care. Resuscitation 2008;79(1):61-6.

21. Cudnik MT, Schmicker RH, Vaillancourt C, Newgard CD, Christenson JM, Davis DP, et al. A geospatial assessment of transport distance and survival to discharge in out of hospital cardiac arrest patients: Implications for resuscitation centers. Resuscitation 2010;81 (5):518-23.

22. Kajino K. Impact of transport to critical care medical centers on outcomes after out-of-hospital cardiac arrest. Resuscitation. 2010;In Press.

23. Birkmeyer JD, Stukel TA, Siewers AE, Goodney PP, Wennberg DE, Lucas FL. Surgeon volume and operative mortality in the United States. N Engl J Med 2003;349(22):2117-27.

24. Carr BG, Goyal M, Band RA, Gaieski DF, Abella BS, Merchant RM, et al. A national analysis of the relationship between hospital factors and post-cardiac arrest mortality. Intensive Care Med 35(3):505-11.

25. Nathens AB, Jurkovich GJ, Maier RV, Grossman DC, MacKenzie EJ, Moore M, et al. Relationship between trauma center volume and outcomes. JAMA 2001;285(9):1164-71. 
26. Canto JG, Every NR, Magid DJ, Rogers WJ, Malmgren JA, Frederick PD, et al. The volume of primary angioplasty procedures and survival after acute myocardial infarction. The National Registry of Myocardial Infarction 2 Investigators. N Engl J Med 2000;342(21):1573-80.

27. Kahn JM, Goss CH, Heagerty PJ, Kramer AA, O'Brien CR, Rubenfeld GD. Hospital volume and the outcomes of mechanical ventilation. N Engl J Med 2006;355(1):41-50

28. Callaway CW, Schmicker RH, Kampmeyer M, Powell J, Nichol G, Rea TD, et al. Influence of Receiving Hospital Characteristics on Survival after Cardiac Arrest ŠAbstractĆ. Circulation 2008;118(S):1446.

29. Nichol G, Aufderheide TP, Eigel B, Neumar RW, Lurie KG, Bufalino VJ, et al. Regional systems of care for out-of-hospital cardiac arrest: A policy statement from the American Heart Association. Circulation 2010;121(5):709-29.

30. Dalby M, Bouzamondo A, Lechat P, Montalescot G. Transfer for primary angioplasty versus immediate thrombolysis in acute myocardial infarction: a meta-analysis. Circulation 2003;108(15):1809-14.

31. Antman EM, Hand M, Armstrong PW, Bates ER, Green LA, Halasyamani LK, et al. 2007 focused update of the ACC/AHA 2004 Guidelines for the Management of Patients With ST-Elevation Myocardial Infarction: a report of the American College of Cardiology/American Heart Association Task Force on Practice Guidelines: developed in collaboration with the Canadian Cardiovascular Society, endorsed by the American Academy of Family Physicians: 2007 Writing Group to Review New Evidence and Update the ACC/AHA 2004 Guidelines for the Management of Patients With ST-Elevation Myocardial Infarction, writing on behalf of the 2004 Writing Committee. Circulation 2008;117(2):296-329.

32. Mongan JJ, Ferris TG, Lee TH. Options for slowing the growth of health care costs. N Engl J Med 2008;358(14):1509-14.

33. Frandsen F, Nielsen JR, Gram L, Larsen CF, Jorgensen HRI, Hole P, et al. Evaluation of intensified prehospital treatment in out-of-hospital cardiac arrest: survival and cerebral prognosis. The Odense Ambulance Study. Cardiology 1991;79:256-64.

34. Bergner L, Bergner M, Hallstrom AP, Eisenberg MS, Cobb LA. Service factors and health status of survivors of out-of-hospital cardiac arrest. Am J Emerg Med 1983;1(3):259-63.

35. Nichol G, Stiell IG, Hebert P, Wells GA, Vandemheen K, Laupacis A. What is the quality of life of survivors of cardiac arrest? A prospective study. Acad Emerg Med 1999;6:95-102.

36. Nichol G, Huszti E, Birnbaum A, Mahoney B, Weisfeldt M, Travers A, et al. Cost-effectiveness of lay responder defibrillation for out-ofhospital cardiac arrest. Ann Emerg Med 2009; In Press

37. Committee on the Future of Emergency Care in the United States. Emergency Medical Services: At the Crossroads. Washington, DC: Institute of Medicine/National Academies Press2006.

38. US Trauma Center Crisis: Lost in the Scramble for Terror Resources. Irvine, CA: National Foundation for Trauma Care; 2004.

39. Fisher A, Ross C, Henderson C, Kirk S, Feroze M, Richmond R, et al. Major Trauma Care in England. London: National Audit Office 2010.

40. Committee on the Future of Emergency Care in the United States. Hospital-Based Emergency Care: At the Breaking Point. Washington, DC: Institute of Medicine/National Academies Press; 2006

41. Lewin ME, Altman S. America's Health Care Safety Net: Intact but Endangered. Washington, DC: Institute of Medicine 2000.

42. Committee on the Future of Emergency Care in the United States Health System Board on Health Care Services. Emergency Medical Services At the Crossroads. Washington, DC: Institute of Medicine, National Academy of Science 2006. 